\title{
PRINCIPAIS DIAGNÓSTICOS DE ENFERMAGEM EM PACIENTES HOSPITALIZADOS PÓS CIRURGIA CARDÍACA: REVISÃO INTEGRATIVA
}

Guilhermina Mara da Silva, Vanessa Alves da Silva. Principais diagnósticos de enfermagem em pacientes hospitalizados pós cirurgia cardíaca: revisão integrativa. Revista Saúde Dinâmica, vol. 3, núm. 2, 2021. Faculdade Dinâmica do Vale do Piranga. 


\title{
Principais diagnósticos de enfermagem em pacientes hospitalizados pós cirurgia cardíaca: revisão integrativa
}

\author{
Main nursing diagnoses in hospitalized patients after cardiac surgery: integrative review
}

\author{
Guilhermina Mara da Silva', Vanessa Alves da Silva ${ }^{2}$ \\ ${ }^{I}$ Discente do Curso de Enfermagem, Faculdade Dinâmica do Vale do Piranga, https://orcid.org/0000-0002-5653-834X \\ ${ }^{2}$ Docente e coordenadora do Curso de Enfermagem, Faculdade Dinâmica do Vale do Piranga, https://orcid.org/0000- \\ 0003-3898-049X \\ Autor correspondente: guilhermina_mara@yahoo.com
}

\section{Resumo}

As doenças cardiovasculares são responsáveis por 30\% de óbitos no Brasil e são consideradas um problema de saúde pública. A sua abordagem varia entre conduta clínica ou cirúrgica, sendo o profissional enfermeiro importante no cuidado a este paciente. Relacionado a conduta cirúrgica, o enfermeiro tem a responsabilidade no planejamento e assistência em todo período perioperatório, realizando a sistematização da assistência de enfermagem de forma eficaz. $\mathrm{O}$ trabalho tem como intuito descrever os principais diagnósticos de enfermagem em pacientes hospitalizados pós cirurgia cardíaca segundo a literatura. Para tal, foram realizados uma revisão integrativa na base de dados da Biblioteca Virtual em Saúde - BVS, utilizando como descritores "diagnóstico de enfermagem", "cirurgia torácica", "assistência perioperatória" e como operador boleano para busca foi empregado o AND. Foram obtidos na busca 28 artigos e após filtros e critérios de inclusão e exclusão, foram eliminados 26, sendo selecionados dois (2) artigos. Para análise dos artigos selecionados, foram contabilizados os diagnósticos de risco alto e foram estabelecidas as intervenções de enfermagem para cada um deles. Destaca-se que alguns diagnósticos foram prevalentes em ambos os artigos, demonstrando a necessidade de pesquisas na área para que o cuidado de enfermagem seja realizado de forma eficaz e com qualidade.

Palavras-chave: Diagnóstico de enfermagem; assistência perioperatória; cirurgia torácica.

\begin{abstract}
Cardiovascular diseases are responsible for $30 \%$ of deaths in Brazil and are considered a public health problem. Its approach varies between clinical and surgical conduct, with the professional nurse being important in the care of this patient. Related to surgical conduct, the nurse is responsible for planning and assistance throughout the perioperative period, performing the systematization of nursing care effectively. The work aims to describe the main nursing diagnoses in hospitalized patients after cardiac surgery according to the literature. To this end, an integrative review was carried out in the database of the Virtual Health Library - BVS, using as descriptors "nursing diagnosis", "thoracic surgery", "perioperative care" and the AND as Boolean operator for search was used. 28 articles were obtained in the search and after filters and inclusion and exclusion criteria, 26 were eliminated, and two (2) articles were selected. To analyze the selected articles, high-risk diagnoses were counted and nursing interventions were established for each one of them. It is noteworthy that some diagnoses were prevalent in both articles, demonstrating the need for research in the area so that nursing care is carried out effectively and with quality.

Key words: Nursing diagnosis; perioperative care; thoracic surgery.
\end{abstract}




\section{INTRODUÇÃO}

Segundo a Sociedade Brasileira de Cardiologia, as doenças cardiovasculares são responsáveis por 30\% de óbitos no Brasil anualmente e são consideradas um problema de saúde pública (SBC, 2019).

São caracterizadas como uma das principais causas de mortalidade mundial e que leva á baixa qualidade de vida por promover incapacidade no indivíduo, estando entre as doenças crônicas não transmissíveis que mais matam no Brasil (LARA, 2017).

Os tratamentos de doenças cardiovasculares variam entre abordagem clínica ou cirúrgica, e a indicação cirúrgica geralmente ocorre quando o paciente apresenta uma cronicidade da doença, onde o tratamento clínico não é efetivo a longo prazo (PIVOTO, 2010).

A cirurgia cardiovascular tem como objetivo resgatar a funcionalidade da bomba cardíaca minimizando os sintomas, fazendo com que o paciente restabeleça a sua capacidade e autonomia. Esse procedimento é considerado de grande porte, podendo ter três tipos de objetivos: correção, reconstrução e substituição (MELO et al, 2018).

Vale ressaltar que o aumento das doenças isquêmicas cardiovasculares influenciou o número grande de estudos na área, onde visam o aperfeiçoamento de técnicas e tratamentos, para melhoria da abordagem cirúrgica, diagnóstica e preventiva. Quando se fala em abordagem cirúrgica, são considerados procedimentos complexos, onde todas as fases operatórias devem ser acompanhadas requerendo um tratamento em cada uma delas (RIBEIRO, 2019).

As fases operatórias são classificadas em pré, trans e pós-operatório, sendo necessário um acompanhamento em cada fase. O pós-operatório das cirurgias cardíacas deve ser acompanhado por todos os profissionais de saúde, pois é considerado o período mais crítico do tratamento cirúrgico. É caracterizado pelo período do qual se acompanha a recuperação do paciente em pós-anestésico e em pós-estresse cirúrgico, onde é marcado pela instabilidade do seu quadro clínico, sendo cheio de peculiaridades (RIBEIRO, 2019).

Por esse motivo, o profissional enfermeiro assume um papel importante no cuidado ao paciente. Tem responsabilidade no planejamento e assistência de forma individual, visando não somente o período pós-operatório, como todo o período perioperatório realizando a assistência de forma eficaz (MELO et al, 2018). 
O enfermeiro deve realizar a sistematização da assistência de enfermagem (SAE) de forma criteriosa, metodológica e planejada, baseada em conhecimento científico, afim de conduzir a equipe quanto ao cuidado ao paciente (MELO et al, 2018).

A SAE é dividida em fases: histórico de enfermagem, diagnóstico de enfermagem, planejamento de enfermagem, intervenção de enfermagem e avaliação de enfermagem. $\mathrm{O}$ diagnóstico de enfermagem constitui-se por uma das etapas privativas do enfermeiro e caracteriza-se pelo reconhecimento dos atuais problemas e os eventuais, para assim oferecer um cuidado com qualidade. Consiste em avaliações clínicas que são executadas pelo enfermeiro, baseando-se nas respostas as diversas situações relacionadas a saúde vivenciadas pelo individuo, a fim de promover intervenções e alcançar metas na assistência prestada (MELO et al, 2019).

Atender o paciente em pós cirurgia cardíaca requer do enfermeiro um julgamento clínico e um raciocínio lógico para que realize um atendimento com segurança, livre de danos e com qualidade. Para tal, questiona-se: Quais são os principais diagnósticos e intervenções de enfermagem em pacientes hospitalizados pós cirurgia cardíaca?

\section{JUSTIFICATIVA}

Diversos estudos apontam a dificuldade de enfermeiros frente a aplicação da SAE nos ambientes de saúde. A principal causa destacada está relacionada a falta de conhecimentos básicos e teóricos para sua aplicação prática, justificando a importância de abordar temas sobre a SAE (TAKAHASHI, 2008).

Uma das fases com maior dificuldade, por ter que realizar julgamento clínico e raciocínio lógico é o diagnóstico de enfermagem. Ele tem fundamental importância quando se trata da assistência que será prestada ao paciente conforme as suas necessidades, o presente estudo objetivou descrever os principais diagnósticos de enfermagem ao paciente pós cirurgia cardíaca, afim de estabelecer intervenções de enfermagem frente aos diagnósticos encontrados e facilitar a percepção do profissional de enfermagem diante desse paciente. 


\section{DESENVOLVIMENTO \\ REFERENCIAL TEÓRICO}

\section{Cirurgias cardíacas}

As cirurgias cardíacas podem ser caracterizadas como corretoras, relacionadas à correção de problemas no canal arterial, ventricular ou septo atrial, podem ser também reconstrutoras designadas a revascularização do músculo cardíaco (miocárdio), ou troca valvar que incluem as plastias das válvulas cardíacas e substitutivas (PIVOTO, 2010).

O músculo cardíaco demanda grande quantidade de oxigênio e nutrientes para sua manutenção, do qual as artérias coronárias são responsáveis. A cirurgia de revascularização do miocárdio aborda as coronárias, instaurando o fornecimento de sangue ideal ao miocárdio (FERNANDES, 2008).

As cirurgias de trocas valvares compreendem na substituição da valva ineficiente por prótese mecânica ou biológica. A mais utilizada atualmente é a valva biológica que pode ser oriunda de tecidos bovinos ou porcinos, por outro lado, próteses mecânicas são produzidas industrialmente e possuem uma durabilidade maior que as valvas biológicas (LARA, 2017).

As cirurgias cardíacas sofreram grandes avanços tecnológicos ao longo dos anos, e juntamente o surgimento da circulação extracorpórea (CEC) que compreende em um conjunto de aparelhos, máquinas, circuitos e técnicas do qual substituem por um determinado tempo as funções do coração e do pulmão, que durante esse período ficam sem circulação, facilitando a abordagem do cirurgião para examinar detalhadamente o interior do coração (SOUZA, 2006).

\section{Enfermeiro na SAE}

Os cuidados de enfermagem são essenciais para a reabilitação do indivíduo submetido a qualquer um desses procedimentos cirúrgicos, por isso, a Sistematização da Assistência de Enfermagem (SAE) vem se aperfeiçoando juntamente com as técnicas cirúrgicas afim de oferecer novas estratégias de assistência de forma individual que atendam tanto o paciente quanto aos familiares (LARA, 2017).

A SAE é composta por cinco fases que são, o histórico de enfermagem, os diagnósticos de enfermagem, o planejamento, a implementação e a avaliação dos resultados (RIBEIRO, 2019). 
Conforme a resolução do COFEN-358/2009, o diagnóstico de enfermagem é um processo de interpretação e junção dos dados coletados que finda na tomada de decisão sobre os diagnósticos que melhor representem as respostas do indivíduo, família ou coletividade em algum momento do processo saúde-doença, que compõem uma base para selecionar as ações e intervenções de enfermagem afim de alcançar bons resultados (COFEN, 2009).

A Taxonomia mais usada para traçar os diagnósticos de enfermagem é o NANDA, contendo 244 diagnósticos de enfermagem que estão classificados em 13 domínios e distribuídos dentro de cada classe (NANDA, 2018-2020).

O enfermeiro realiza diversas intervenções voltadas para o cuidado nas diversas fases do período perioperatório, mais especificamente no pós-operatório imediato que constitui as primeiras 24 horas após o procedimento cirúrgico (LARA, 2017).

\section{METODOLOGIA}

\section{Tipo de estudo}

A metodologia utilizada neste trabalho foi através de uma pesquisa bibliográfica que, de acordo com Gil (2010, p. 29), é elaborada com base em material já publicado como: material impresso, livros, revistas, jornais, teses, dissertações e anais de eventos científicos.

Segundo Gil (2010) a revisão de literatura é composta das seguintes fases: delimitação da questão problema ou hipótese, estabelecimento dos critérios para a seleção da pesquisa, análise crítica e interpretação dos resultados e apresentação da revisão.

\section{Pergunta de pesquisa}

A pergunta que norteou a pesquisa foi: Quais são os principais diagnósticos e intervenções de enfermagem em pacientes hospitalizados pós cirurgia cardíaca?

\section{Estratégia de busca}

A estratégia de busca das produções cientificas foi realizada na base de dados Biblioteca Virtual de Saúde - BVS. Buscou-se apresentar uma revisão narrativa da literatura, através da busca eletrônica de artigos em periódicos brasileiros. 
O período de coleta dos artigos foi no mês de julho de 2020. Como critérios de inclusão foram considerados artigos na íntegra, que abordassem o tema diagnóstico de enfermagem em pós de cirurgia cardíaca e foram excluídos os artigos pagos.

Como descritores para busca dos artigos foram considerados: "diagnóstico de enfermagem", "período perioratório" e "cirurgia torácica". Como operador boleano para busca foi utilizado o AND.

\section{Coleta de Dados}

Foi utilizada a base de dados da Biblioteca Virtual em Saúde - BVS. Os descritores considerados para busca dos artigos foram: diagnóstico de enfermagem, cirurgia torácica e período perioperatório e como operador boleano para busca foi empregado o AND.

Figura 1- Descrição da busca dos artigos

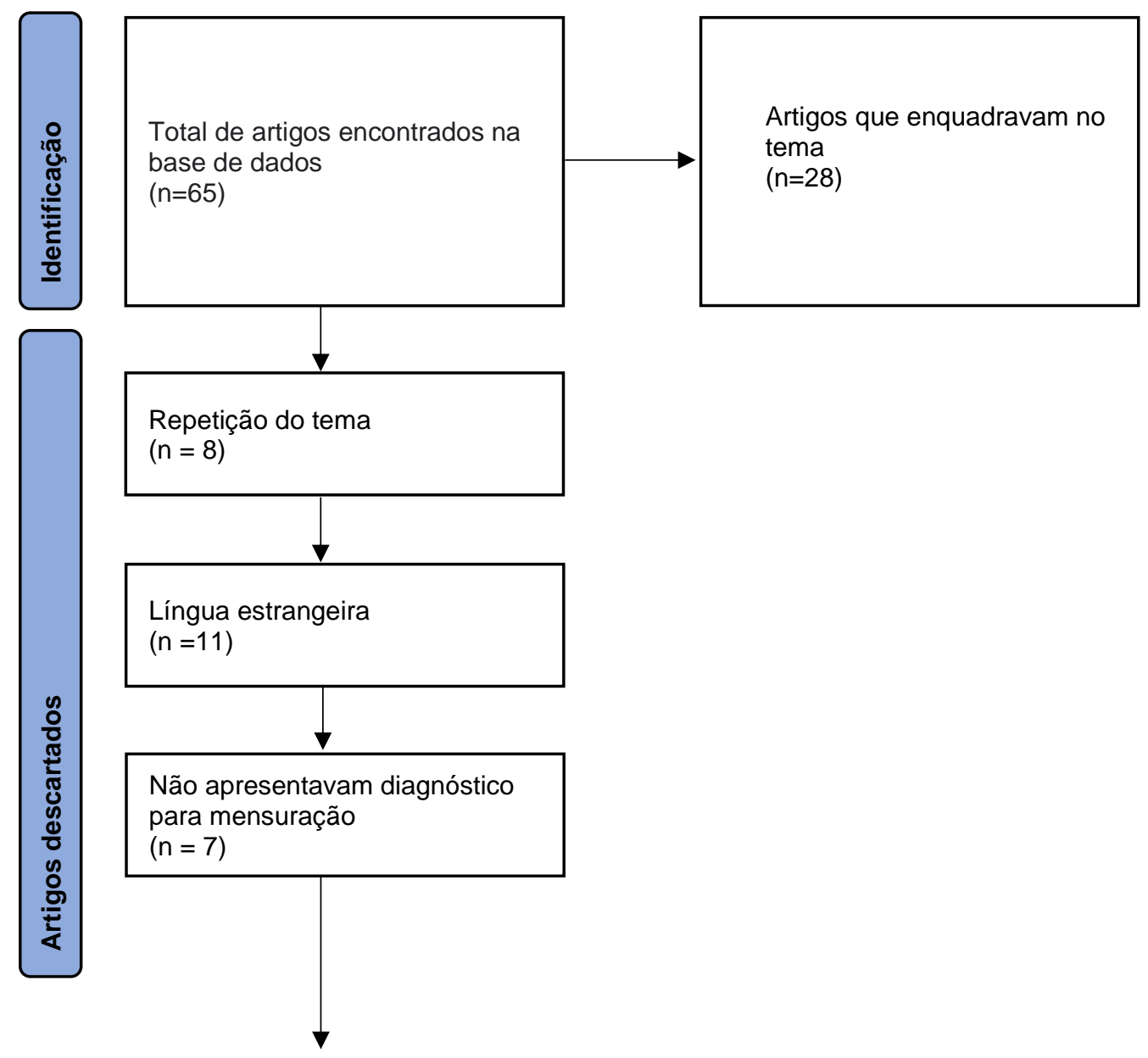


Total de artigos selecionados $(\mathrm{n}=2)$

Após leitura dos 28 artigos, foram excluídos 26 devido repetição do tema, língua estrangeira, não apresentavam diagnósticos para mensuração, sendo selecionados dois (2) artigos.

\section{Análise}

Foi utilizado um instrumento de coleta de dados, elaborado pela pesquisadora para avaliação das publicações selecionadas. Ele contempla os itens: título, objetivos, local de publicação e principais resultados.

Os estudos foram explorados e os dados digitados em banco de dados do Microsoft Excel 2013 para análise estatística descritiva. Em seguida, foram difundidos em tabelas para a síntese das informações dos periódicos.

\section{RESULTADO E DISCUSSÃO}

Para desenvolvimento do trabalho foram selecionados artigos de diagnóstico de enfermagem em pacientes que foram submetidos a cirurgia cardíaca no período de pósoperatório.

Na tabela abaixo foram descritas informações como: título do artigo, o objetivo do artigo, o local de publicação ou revista e os resultados, onde estão detalhados em número os diagnósticos de enfermagem encontrados. 
Tabela 1: diagnósticos de enfermagem encontrados nos artigos

\begin{tabular}{|c|c|c|c|}
\hline Título & Objetivo & $\begin{array}{c}\text { Local de } \\
\text { publicação ou } \\
\text { revista }\end{array}$ & Resultados \\
\hline $\begin{array}{c}\text { Diagnósticos de } \\
\text { enfermagem no pós } \\
\text { operatório imediato de } \\
\text { cirurgia de troca de } \\
\text { válvula }\end{array}$ & $\begin{array}{c}\text { Identificar os } \\
\text { diagnósticos de } \\
\text { enfermagem mais } \\
\text { frequentes nos pós } \\
\text { operatórios imediato } \\
\text { de cirurgia de troca } \\
\text { de válvula realizada } \\
\text { em } 50 \text { pacientes } \\
\text { (LARA, 2017). }\end{array}$ & $\begin{array}{l}\text { Revista de } \\
\text { Enfermagem } \\
\text { UFSM 2017 }\end{array}$ & $\begin{array}{l}\text { Serão descritos os } \\
\text { diagnósticos de } \\
\text { enfermagem } \\
\text { encontrados no artigo: } \\
\text { Risco baixo volume - } \\
41 \\
\text { Locomoção } \\
\text { prejudicada - } 47 \\
\text { Força cardíaca - } 32 \\
\text { Dificuldade em } \\
\text { comunicar - } 28 \\
\text { Relações familiares } \\
\text { cortadas - } 28 \\
\text { Risco de adquirir } \\
\text { infecção - 50 } \\
\text { Risco de cair - } 47 \\
\text { Risco de lesão em } \\
\text { pele - } 46 \\
\text { Lesão em pele - } 44 \\
\text { Risco de aspiração - } \\
36 \\
\text { Temperatura baixa - } \\
10\end{array}$ \\
\hline $\begin{array}{c}\text { Diagnósticos de } \\
\text { enfermagem a pacientes } \\
\text { submetidos a cirurgia } \\
\text { cardíaca }\end{array}$ & $\begin{array}{l}\text { Identificação dos } \\
\text { diagnósticos de } \\
\text { enfermagem, } \\
\text { segundo a taxonomia } \\
\text { II a NANDA para o } \\
\text { planejamento da } \\
\text { assistência de } \\
\text { enfermagem em } \\
\text { pacientes no pós } \\
\text { operatório de } \\
\text { cirurgia cardíaca de }\end{array}$ & $\begin{array}{l}\text { Revista Brasileira } \\
\text { de Enfermagem, } \\
2006\end{array}$ & $\begin{array}{l}\begin{array}{l}\text { Serão descritos os } \\
\text { diagnósticos } \\
\text { enfermagem } \\
\text { encontrados no artigo: }\end{array} \\
\text { Risco de adquirir } \\
\text { infecção - } 22 \\
\text { Risco de constipação } \\
-22\end{array}$ \\
\hline
\end{tabular}




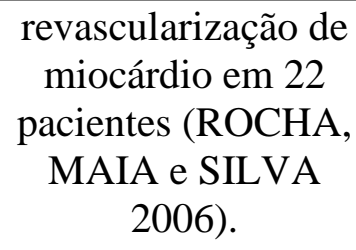

\author{
Não consegue realizar \\ atividades de \\ autocuidado para \\ vestir-se- 22 \\ Lesão em pele - 22 \\ Locomoção \\ prejudicada - 20 \\ Integridade da pele \\ prejudicada - 20 \\ Não consegue realizar \\ atividades de \\ autocuidado para \\ comer- 15
}

Sono alterado -15

Padrão respiratório ineficaz - 15

Ansiedade - 15

Desobstrução ineficaz de vias aéreas - 14

Dor aguda - 13

Nutrição ingerida menos que as necessidades -11

Ventilação espontânea prejudicada - 11

Risco de não tolerar a atividade -11

Risco para aspiração 6

Fala prejudicada - 6

Baixo volume - 4 
Rocha, Maia e Silva (2006) estabeleceram seus diagnósticos a partir da cirurgia de revascularização de miocárdio, e Lara (2017) determinou os diagnósticos citados com base no pós-operatório da cirurgia de troca valvar.

Ambas cirurgias citadas são complexas, de grande porte e com o pós-operatório crítico, devendo o enfermeiro realizar uma assistência eficaz e eficiente.

De acordo com Lara (2017) na cirurgia de troca valvar a prótese biológica é utilizada com mais frequência por isentar o uso de anticoagulantes de maneira contínua e ininterrupta após a cirurgia, entretanto, existem algumas limitações relacionadas ao uso desse tipo de prótese, que podem ser, durabilidade, calcificações, risco cirúrgico e podendo haver a necessidade de reoperações. Por outro lado, as próteses mecânicas não apresentam desgaste com o tempo, contudo, é imprescindível o controle da coagulação.

Os pacientes que realizam cirurgias cardiovasculares são caracterizados por terem uma condição de saúde do qual padecem de mudanças contínuas e abruptas, esses tipos de procedimentos necessitam de cuidados de enfermagem que são essenciais para a sua recuperação (ROCHA, MAIA E SILVA, 2006).

Diante do objetivo de estabelecer os principais diagnósticos de enfermagem em pacientes no pós-operatório de cirurgia cardíaca, a pesquisadora realizou uma classificação de riscos onde os diagnósticos de enfermagem encontrados foram especificados como risco baixo, risco moderado e risco alto. Essa classificação deu-se através de uma média de diagnósticos encontrados nos pacientes.

Dessa forma as intervenções de enfermagem foram traçadas apenas para os diagnósticos classificados como risco alto. A Taxonomia usada para traçar as intervenções de enfermagem foi a NIC - Classificação das Intervenções de Enfermagem, $6^{\circ}$ edição, conforme descrito na tabela abaixo.

Tabela 2- Diagnósticos e intervenções de enfermagem no pós-operatório imediato de cirurgia de troca de válvula
Diagnósticos de risco alto
Intervenções de enfermagem

Monitorização de eletrólitos;

Controle hidroeletrolítico;

Risco de volume de líquido deficiente Controle hídrico; 


\begin{tabular}{|c|c|}
\hline & $\begin{array}{l}\text { Proteção contra infecção; } \\
\text { Punção venosa } \\
\text { Controle de choque; } \\
\text { Reposição volêmica; }\end{array}$ \\
\hline Mobilidade física prejudicada & $\begin{array}{l}\text { Controle da dor; } \\
\text { Controle do ambiente; } \\
\text { Cuidados com o repouso no leito; } \\
\text { Posicionamento; } \\
\text { Controle da sensibilidade periférica; } \\
\text { Prevenção contra quedas; }\end{array}$ \\
\hline Débito cardíaco diminuído & $\begin{array}{l}\text { Monitorização acidobásica; } \\
\text { Monitorização de eletrólitos; } \\
\text { Monitorização de sinais vitais; } \\
\text { Oxigenoterapia; } \\
\text { Prevenção de choque; }\end{array}$ \\
\hline Comunicação verbal prejudicada & $\begin{array}{l}\text { Intermediação cultural; } \\
\text { Presença; } \\
\text { Redução da ansiedade; } \\
\text { Toque; } \\
\text { Redução do estresse por mudança; }\end{array}$ \\
\hline Processos familiares interrompidas & Promoção do envolvimento familiar; \\
\hline Risco de infecção & $\begin{array}{l}\text { Prevenção de ulceras por pressão; } \\
\text { Cuidados com o local da incisão; } \\
\text { Promoção da saúde oral; } \\
\text { Controle de vias aéreas; } \\
\text { Cuidados com drenos torácicos; } \\
\text { Controle hidroeletrolítico; } \\
\text { Cuidados com sondas urinárias; } \\
\text { Monitorização de sinais vitais; }\end{array}$ \\
\hline
\end{tabular}




\section{Risco de queda}

\begin{tabular}{ll} 
& Posicionamento; \\
& Restrição de área; \\
& Posicionamento; \\
& Identificação de risco; \\
& Assistência no autocuidado; \\
\hline Risco de integridade da pele & Cuidados com o local de incisão; \\
prejudicada & Monitorização das \\
& inferiores; \\
& Posicionamento; \\
& Prevenção de ulceras por pressão; \\
& Cuidados com a tração/imobilização; \\
& Cuidados com o local de incisão; \\
& Cuidado com sondas urinárias; \\
\hline prejudicada & Monitorização respiratória; \\
& Controle da ventilação mecânica invasiva; \\
& Desmame da ventilação mecânica; \\
Misco de aspiração & Monitorização dos sinais vitais (saturação \\
& de O²); \\
\hline &
\end{tabular}

Fonte: Elaborada pelos autores com base em Lara, 2017

Os dados levantados na primeira tabela representam as principais necessidades afetadas dos pacientes nas primeiras 24 horas do pós-operatório de cirurgias cardíacas e as intervenções de enfermagem onde demonstram o desafio na assistência prestada ainda na UTI.

As intervenções de enfermagem encontradas direcionam o profissional para um cuidado individualizado e qualificado, a esse paciente que se encontra em um período crítico marcado pela instabilidade do seu quadro (RIBEIRO, 2019).

Na tabela abaixo foram descritos os diagnósticos de enfermagem classificados pela pesquisadora como de rico alto, e as intervenções de enfermagem para cada diagnóstico selecionado. 
Tabela 3- Diagnósticos de enfermagem a pacientes submetidos a cirurgia cardíaca

Diagnósticos de risco alto

Risco de infecção

\section{Intervenções de enfermagem}

Prevenção de ulceras por pressão;

Cuidados com o local da incisão;

Promoção da saúde oral;

Controle de vias aéreas;

Cuidados com drenos torácicos;

Controle hidroeletrolítico;

Cuidados com sondas urinárias;

Monitorização de sinais vitais;

\begin{tabular}{ll}
\hline Risco de constipação & Controle da nutrição; \\
& Controle de medicamentos; \\
& Monitorização nutricional; \\
\hline Déficit no autocuidado para vestir-se & Assistência no autocuidado; \\
& Redução da ansiedade; \\
\hline Integridade da pele prejudicada & Supervisão da pele (sutura); \\
& Cuidados com tração/imobilização; \\
\hline Mobilidade física prejudicada & Controle da dor; \\
& Controle do ambiente; \\
& Cuidados com o repouso no leito; \\
& Posicionamento; \\
& Controle da sensibilidade periférica; \\
& Prevenção contra quedas; \\
& Controle da pressão; \\
& Cuidados com o local de incisão; \\
& Cuidados com sondas urinárias; \\
& Cuidados com drenos; \\
\hline Integridade tissular prejudicada & \\
&
\end{tabular}

Fonte: Elaborada pelos autores com base em Rocha, Maia e Silva 2006. 
Ao estabelecer os diagnósticos de enfermagem mais prevalentes em pacientes hospitalizados pós cirurgia cardíaca verificou-se a necessidade de um direcionamento da assistência de enfermagem que possibilite o cuidado do enfermeiro de qualidade, traçando os diagnósticos e intervenções corretos.

Esse instrumento visa direcionar o processo de enfermagem por meio de teorias e conhecimento cientifico podendo ser visto como uma metodologia que colabora para a implementação da ciência a prática (PIVOTO, 2010).

\section{CONCLUSÃO}

A pesquisa evidenciou os principais diagnósticos de enfermagem prevalentes em ambos os artigos. Foram encontrados dez (10) diagnósticos de enfermagem relacionados à cirurgia de troca de válvula e seis (6) diagnósticos de enfermagem relacionado a cirurgia cardíaca. Quando associado os artigos, verificou-se que somente um diagnóstico apareceu igual em ambos.

A pesquisa demonstra que o enfermeiro executa um papel primordial no atendimento ao paciente e deve realizar seu cuidado de forma sistematizada e com planejamento. A sistematização da assistência de enfermagem norteia o enfermeiro em sua conduta, portanto, conhecer os diagnósticos mais prevalentes em pós-operatório cardíaco e determinar as principais intervenções auxilia no cuidado prestado por este profissional.

Ressalta-se a necessidade de mais pesquisas na área no intuito de melhorar a conduta prestada pelo enfermeiro.

\section{REFERÊNCIAS}

Lara, Bruna Fontes de; NOGUEIRA, Paula Cristina; POVEDA, Vanessa de Brito. Diagnósticos de enfermagem no pós operatório imediato de cirurgia de troca de válvula. Revista de Enfermagem da UFSM, [S. 1.], p. 700-711, 15 dez. 2017. Disponível em: https://periodicos.ufsm.br/reufsm/article/view/25716. Acesso em: 13 maio 2020.

Melo FV, Costa MF, Sandes SMS. Diagnósticos de Enfermagem no Período Pós-Operatório de Cirurgia Cardíaca. Rev Rev enferm UFPE online., Recife, 12(8):2188-93, ago., 2018. 1981-8963. 
Nanda International. Diagnósticos de enfermagem da NANDA-I: definições e classificação 2018-2020. 11. ed. Porto Alegre: Artmed, 2018.

Pivoto FL, Lunardi Filho WD, Santos SSC, et al. Diagnósticos de enfermagem em pacientes no período pósoperatório de cirurgias cardíacas. Acta Paul Enferm 2010;23(5):665-70.

Mai/Jun 2010.

Ribeiro KRA, Gonçalves FAF, Borges MM, et al. Pós-Operatório de Revascularização do Miocárdio: Possíveis Diagnósticos e Intervenções de Enfermagem. Rev Fund Care Online. abr./jun, 2019.

Rocha Luciana Alves da; MAIA, Ticiane Fernandes; SILVA, Lúcia de Fátima da. Diagnósticos de enfermagem em pacientes submetidos à cirurgia cardíaca. Revista Brasileira de Enfermagem, [S. 1.], p. 323-324, 1 jun. 2006. Disponível em:

https://www.scielo.br/scielo.php?pid=S0034-

71672006000300013\&script=sci_abstract\&tlng=pt. Acesso em: 12 maio 2020.

Souza, M.H.L. Elias, D.O. Fundamentos da Circulação Extracorpórea. Centro Editorial Alfa Rio. Rio de Janeiro, v.2, p.33, 2006

Takahashi, Alda Akie et al. Dificuldades e facilidades apontadas por enfermeiras de um hospital de ensino na execução do processo de enfermagem. Acta paul. enferm. São Paulo,v. 21, n. 1, p. 32-38,Mar.2008

\section{Declaração de Interesse}

Os autores declaram não haver nenhum conflito de interesse

\section{Financiamento}

Financiamento próprio

\section{Agradecimentos}

Grata primeiramente á Deus, pela minha vida e por me ajudar a ultrapassar todos os obstáculos ao longo da graduação.

Aos meus pais, João Batista da Silva e Maria da Conceição Silva e irmãos, dos quais em meio há tantas dificuldades sempre estiveram ao meu lado. A minha querida orientadora Prof ${ }^{o}$ Vanessa Alves da Silva, por me permitir apresentar um melhor desempenho no meu processo de formação profissional.

\author{
Colaboração entre autores \\ O presente artigo foi escrito pela G.M.S \\ sob orientação do professor V.A.S., \\ projetado e concluído no Programa \\ Institucional de Bolsas de Iniciação \\ Científica (PIBIC) da Faculdade \\ Dinâmica do Vale do Piranga (FADIP). \\ Ambos os autores cuidaram da parte \\ dissertativa do artigo.
}

